\title{
Práticas de autocuidado dos portadores de diabetes mellitus tipo II: contribuições da teoria de Dorothea Orem
}

\author{
Self-care practices of people with type II diabetes mellitus: contributions from Dorothea \\ Orem's theory
}

Prácticas de autocuidado de las personas con diabetes mellitus tipo II: aportaciones de la
teoría de Dorothea Orem

Jackelliny Carvalho Neves ${ }^{1 *}$, Luciane Sousa Pessoa Cardoso ${ }^{1}$, Andressa Arraes Silva1, Rafaela Duailibe Soares $^{2}$, Joelmara Furtado dos Santos Pereira ${ }^{2}$, Alan Cássio Carvalho Coutinho², Larissa Silva Oliveira ${ }^{1}$, Maria Beatriz Pereira da Silva1, Railda Lima Rodrigues ${ }^{1}$, Ana Cláudia de Almeida Varão.

\section{RESUMO}

Objetivo: Analisar as práticas de autocuidado dos portadores de diabetes tipo II e as contribuições da teoria de Dorothea Orem. Métodos: Trata-se de um estudo transversal, de caráter descritivo com abordagem quantitativa, desenvolvida em uma Unidade Básica de Saúde de um município do estado do Maranhão. Na qual foram realizadas entrevistas semiestruturadas acerca das dimensões do autocuidado como alimentação, atividade física, monitorização da glicemia e o cuidado com os pés com 60 portadores de diabetes mellitus tipo II, no período de dezembro de 2019. Os dados obtidos foram analisados através do epi info versão 7.2. Resultados: A população predominante foi do sexo feminino $(73,33 \%)$, com idade superior a 60 anos (41,67\%). 53,33\% referiram não conhecer as práticas de autocuidado. No qual possuem baixa adesão no quesito de atividade física, cuidados com os pés, monitorização glicêmica e consultas diárias. Porém apresentando bons resultados quanto alimentação saudável e não ser tabagista. Conclusão: Foi constatado conhecimento ineficaz dos portadores em relação às atividades de autocuidado, baixa adesão no que se refere à prática de atividade física, cuidado com os pés, monitorização glicêmica e consultas diárias. Porém, quanto a uma alimentação saudável e a não serem tabagistas, apresentaram resultados satisfatórios.

Palavras-chave: Enfermagem, Diabetes, Autocuidado, Adesão.

\begin{abstract}
Objective: To analyze the self-care practices of people with type II diabetes and the contributions of Dorothea Orem's theory. Methods: This is a cross-sectional, descriptive study with a quantitative approach, developed in a Basic Health Unit of a municipality in the state of Maranhão. Semi-structured interviews were conducted about the dimensions of self-care such as nutrition, physical activity, blood glucose monitoring and foot care with 60 patients with type II diabetes mellitus in December 2019. The data obtained were analyzed using the epi info version 7.2. Results: The predominant population was female (73.33\%), aged over 60 years $(41.67 \%)$. $53.33 \%$ reported not knowing the self-care practices. In which they have low adherence regarding physical activity, foot care, glycemic monitoring and daily consultations. However, they showed good results regarding healthy eating and not being a smoker. Conclusion: It was found ineffective knowledge of patients regarding self-care activities, low adherence to physical activity, foot care, glycemic monitoring and daily consultations. However, as for a healthy diet and not being smokers, they showed satisfactory results.
\end{abstract}

Keywords: Nursing, Diabetes, Self-care, Adherence.

\section{RESUMEN}

Objetivo: Analizar las prácticas de autocuidado de los portadores de diabetes tipo II y las aportaciones de la teoría de Dorothea Orem. Métodos: Se trata de un estudio transversal, descriptivo y con enfoque cuantitativo, desarrollado en una Unidad Básica de Salud de un municipio del estado de Maranhão. Se realizaron entrevistas semiestructuradas sobre las dimensiones del autocuidado, como la nutrición, la actividad física, el

1 Universidade Estadual do Maranhão (UEMA), Bacabal - MA. *E-mail: jack_carvalho10@hotmail.com

2 Universidade Federal do Maranhão (UFMA), São Luís - MA. 
control de la glucemia y el cuidado de los pies, a 60 pacientes con diabetes mellitus tipo II durante el periodo de diciembre de 2019. Los datos obtenidos se analizaron con la versión 7.2 de epi info. Resultados: La población predominante fue el sexo femenino (73,33\%), con una edad superior a los 60 años (41,67\%). El $53,33 \%$ declaró no conocer las prácticas de autocuidado. En el que tienen una baja adherencia en cuanto a la actividad física, el cuidado de los pies, el control de la glucemia y las consultas diarias. Porém apresentando bons resultados quanto alimentar saudável e não ser tabagista. Conclusión: Se constató un conocimiento insuficiente de los portadores en relación con las actividades de autocuidado, un bajo nivel de adiestramiento en lo que se refiere a la práctica de la actividad física, el cuidado de los pies, la monitorización glicémica y las consultas diarias. Por lo tanto, en lo que respecta a la alimentación sana y a no ser tabáquico, los resultados son satisfactorios.

Palabras clave: Enfermería, Diabetes, Autocuidado, Adherencia.

\section{INTRODUÇÃO}

A Diabetes Mellitus (DM) é uma doença metabólica de origem multifatorial, decorrente de defeitos na secreção ou ação da insulina. A insulina é um hormônio secretado pelas células beta do pâncreas, que induz o movimento de glicose do sangue para o músculo, o fígado e as células adiposas e seu déficit leva a altos níveis de glicose no sangue (hiperglicemia) e consequentemente a diabetes (HINKLE JL e CHEEVER KH, 2015).

Seu aumento está intrinsicamente associado aos maus hábitos de vida. O que requer mudanças no cotidiano dos portadores, assim como conhecimento de boas práticas de autocuidado para um melhor manejo clínico, como plano alimentar, monitorização glicêmica, atividades físicas, uso correto de medicações e cuidado com os pés. Pois são cuidados necessários para prevenção, tratamento e controle da DM (EID ADD, et al., 2018).

Autocuidado são atividades que os indivíduos realizam em seu próprio benefício para manter um bom estado de saúde, tanto físico como mental. E quando é efetivamente realizado, ajuda a manter a integridade estrutural e o funcionamento do corpo, mas a realização da mesma pode ser afetada por fatores condicionantes básicos, como idade, sexo, estado de saúde, padrões de vida, etc, que influi diretamente na qualidade das práticas de autocuidado (PIRES AF, et al., 2015).

A Teoria de Dorothea E. Orem foi desenvolvida em 1991, e abrange mais três subteorias que estão correlacionadas: a teoria do autocuidado, que relata o porquê e como as pessoas podem cuidar de si; a teoria do déficit do autocuidado, que é a teoria geral, a qual descreve como as pessoas podem ser ajudadas pela enfermagem de acordo com suas dificuldades; e a teoria dos sistemas de enfermagem, que descreve e explica a relação que tem que ser estabelecida para que se produza a enfermagem. Com isso o enfermeiro pois utiliza-se dessa estratégia teórica para a promoção da saúde e prevenção de doenças e suas possíveis complicações, principalmente em patologias crônicas (GEORGE JB, et al., 2000; QUEIRÓS PJP, et al., 2014).

A adoção de tais medidas diariamente por parte dos portadores, é tida como ponto chave para sua terapêutica e prevenção, visto que intervenções no estilo de vida favorecem a eficácia do tratamento e melhoram, consequentemente, a vida diária do indivíduo. Para que possam ser desenvolvidas é necessário que o portador tenha conhecimento e seja apto para desempenhar seu próprio cuidado, que influenciará diretamente na sua autonomia sobre seu estado de saúde (SOUSA JT, et al., 2015).

Pois, o diabetes não cuidado ou tratado de maneira errada leva a várias complicações, o que acarreta gastos elevados para a saúde pública, além do impacto que causa no portador e nos familiares, na qual tem sua rotina alterada em decorrência da incapacidade que a doença apresenta (OLIVEIRA OS, et al., 2017).

Havendo assim, a necessidade de avaliar as práticas de autocuidado dos portadores de DM, possibilitando conhecer o perfil dos mesmos e identificar aqueles com conhecimento ineficaz e com baixa adesão ao tratamento, seja por falta de orientações ou por baixa motivação, assim elaborando um planejamento de acordo com suas necessidades. O presente estudo tem como objetivo analisar as práticas de autocuidado dos portadores de diabetes tipo II e as contribuições da teoria de Dorothea Orem. 


\section{MÉTODOS}

Trata-se de uma pesquisa transversal, de caráter descritivo com abordagem quantitativa, desenvolvida na Estratégia de Saúde da Família (ESF) em um município do estado do Maranhão.

A população pesquisada constituiu-se de 60 portadores de diabetes mellitus tipo II, acompanhados pelas equipes de ESF. Foram incluídos na pesquisa portadores diagnosticado no mínimo a 6 meses, cadastrados na respectiva UBS e que aceitasse a fazer parte do estudo assinando o Termo de Consentimento Livre e Esclarecido (TCLE). Sendo que os critérios de exclusão foram, menos de 6 meses de diagnostico, diagnosticado com diabetes tipo I ou gestacional, que não estivessem cadastrados na respectiva UBS ou que se recusasse a participar da pesquisa.

Os dados foram coletados através de um questionário sobre o respectivo tema com perguntas fechadas. Contendo dados sociodemográficos e sobre as dimensões do autocuidado como alimentação, atividade física, monitorização da glicemia e o cuidado com os pés. O questionário foi aplicado na recepção da UBS, onde os portadores foram informados a respeito das objetivações do trabalho e, da autorização para a realização deste estudo através da assinatura do Termo de Consentimento Livre e Esclarecido.

A análise dos dados ocorreu através do software Epi info versão 7.2, que é uma série de programas desenvolvidos pelo CDC (Centers for Disease Control and Prevention) na qual possui ferramentas que auxiliam na coleta, análise e visualização de dados. E depois foram agrupados em tabelas e gráficos para melhor compreensão.

A pesquisa foi submetida e aprovada de acordo com os princípios éticos e legais do Comitê de Ética em pesquisa com Seres Humanos da Universidade Estadual do Maranhão, campus Caxias, sob parecer 3.784.064.

\section{RESULTADOS}

O estudo foi realizado com 60 portadores de diabetes tipo II, destes, $44(73,33 \%)$ são do sexo feminino e $16(26,67 \%)$ do sexo masculino; a idade mínima foi de 35 anos e a máxima de 94 anos. Em relação à raça, a que obteve maior porcentagem foi a parda com, 31 (51,67\%), seguida pela cor preta, com $18(30,00 \%)$, e a branca, com 11 (18,33\%). Quanto ao estado civil, o maior percentual foi encontrado em indivíduos casados $26(43,33 \%)$ e $6(10 \%)$ em união estável, $14(23,33 \%)$ solteiros e $12(20 \%)$ viúvo. No que diz respeito ao grau de escolaridade, a maioria dos portadores eram analfabetos $24(40 \%), 16(26,67 \%)$, com ensino fundamental incompleto, e apenas $8(13,33 \%) \mathrm{com}$ ensino médio completo. Ao analisar a profissão que exercem, 40 $(66,67 \%)$ eram aposentados e $12(20 \%)$ domésticas, com percentual de até um 1 salário mínimo, havendo um predomínio de 42 (70\%).

Figura 1 - Conhecimento dos portadores de diabetes mellitus tipo Il em relação as atividades de autocuidado.

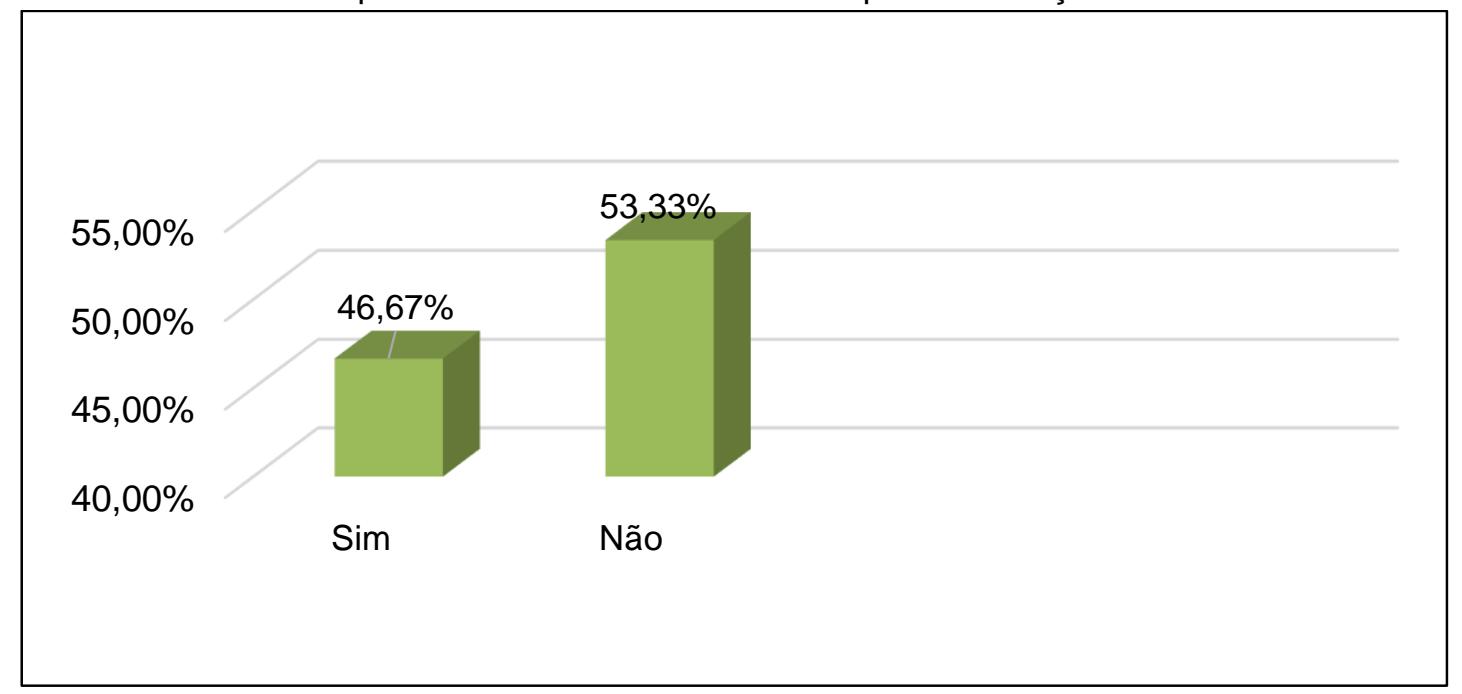

Fonte: Neves JC, et al., 2021. 
Ao investigar o nível de conhecimento dos portadores em relação as atividades de autocuidado, 32 $(53,33 \%)$ referiram não conhecer tais práticas e apenas 28 (46,67\%) conheciam (Figura 1). Mas vale ressaltar que o presente estudo foi composto por pessoas de idade elevada, sendo que a maioria dos indivíduos teve uma formação escolar precária, em decorrência da falta de acesso às escolas e do baixo poder aquisitivo.

Tabela 1 - Frequência, em dias na semana, da prática de atividades físicas, alimentação, saudável, ingestão de gorduras e açúcares e tabagismo dos portadores de diabetes mellitus tipo II.

\section{Frequência}

de dias na

Itens semana

Total

\begin{tabular}{ccccccc} 
& \multicolumn{5}{c}{ semana } & Total \\
\cline { 2 - 5 } & $\mathbf{0 ~ d i a s}$ & $\mathbf{1 ~ v e z}$ & $\mathbf{2 ~ a ~ 3 ~ v e z e s ~}$ & $\mathbf{5}$ vezes & $\begin{array}{c}\text { Mais de 5 } \\
\text { vezes }\end{array}$ & \\
\hline $\begin{array}{c}\text { Praticam } \\
\text { atividade } \\
\text { física }\end{array}$ & $45(75 \%)$ & $4(6,67 \%)$ & $5(8,33 \%)$ & $1(1,67)$ & $5(8,33 \%)$ & $100 \%$ \\
\hline $\begin{array}{c}\text { Segui uma } \\
\text { dieta } \\
\text { saudável }\end{array}$ & $5(8,33 \%)$ & $15(25 \%)$ & $19(31,67 \%)$ & $6(10 \%)$ & $15(25,00 \%)$ & $100 \%$ \\
\hline $\begin{array}{c}\text { Ingeri } \\
\text { gorduras e } \\
\text { açúcares }\end{array}$ & $45(75 \%)$ & $2(3,33 \%)$ & $6(10 \%)$ & 0 & $7(11,67 \%)$ & $100 \%$ \\
\hline $\begin{array}{c}\text { Faz uso do } \\
\text { tabaco }\end{array}$ & $51(85 \%)$ & 0 & 0 & 0 & $9(15 \%)$ & $100 \%$ \\
\hline
\end{tabular}

Fonte: Neves JC, et al., 2021.

O presente estudo observou que 45 (75\%) dos portadores não praticam atividade física e apenas 15 (25\%) praticam. Sendo que a frequência da prática de atividades físicas entre a maioria dos participantes varia de 2 a 3 vezes por semana e mais de 5 vezes na semana (Tabela 1).

Quanto a alimentação $55(91,67 \%)$ dos portadores consome diariamente vegetais, frutas, legumes ou grãos e cinco (8,33\%) não consome; $15(25 \%)$ ingerem diariamente alimentos ricos em gorduras e açúcares e $45(75 \%)$ não ingerem. A maioria mantém uma alimentação saudável 2 a 3 vezes na semana e mais de 5 vezes na semana, porém ingerem gorduras e açúcares com a mesma frequência de dias. Os resultados apresentam comportamentos desejáveis, já que a maioria dos indivíduos referiram ter uma alimentação saudável, sendo que os que não apresentaram uma dieta satisfatória associaram tal fato às baixas condições socioeconômicas ou à falta de orientação pelos profissionais.

No tocante ao tabagismo entre os portadores, 51 (85\%) informou não fumar e apenas nove (15\%) afirmaram-se fumantes, o que apresenta resultados satisfatório.

Tabela 2 - Distribuição dos portadores de diabetes mellitus tipo II que examina seus pés, fazem controle glicêmico e realizam consultas diárias.

\begin{tabular}{ccccccc}
\hline \multicolumn{1}{c}{ Itens } & & Frequência & Total \\
\hline $\begin{array}{c}\text { Examinam } \\
\text { seus pés }\end{array}$ & $\mathbf{0}$ dias & $\begin{array}{c}\text { 1 vez na } \\
\text { semana }\end{array}$ & $\begin{array}{c}\text { 2 a 3 vezes } \\
\text { na semana }\end{array}$ & $\begin{array}{c}\text { 5 vezes na } \\
\text { semana }\end{array}$ & $\begin{array}{c}\text { Mais de 5 vezes } \\
\text { na semana }\end{array}$ & $100 \%$ \\
\hline $\begin{array}{c}\text { Controle } \\
\text { glicêmico }\end{array}$ & Não realiza & Semanal & Mensal & Bimestral & Trimestral & $15 \%)$ \\
\hline $\begin{array}{c}\text { Consultas } \\
\text { diárias }\end{array}$ & $2(3,33 \%)$ & $2(3,33 \%)$ & $15(25 \%)$ & $13(21,67 \%)$ & $28(46,67 \%)$ & $100 \%$ \\
\hline
\end{tabular}

Fonte: Neves JC, et al., 2019. 
No que se refere à autoexaminação diária dos pés, a presente pesquisa aponta que $32(53,33 \%)$ não a realizam e apenas $28(46,67 \%)$ examinam, numa frequência de 2 a 3 vezes na semana e mais de 5 dias na semana em sua maioria, o que apresenta resultado insatisfatório, já que a maioria não realiza essa prática diariamente (Tabela 2).

Foi observado que $58(96,67 \%)$ dos participantes, ou seja, a maioria deles, realizam o controle glicêmico, porém $46,67 \%$ destes o realizam apenas trimestralmente. Quanto aos que realizam a monitorização semanalmente, dava-se ao fato de o portador ter o glicosímetro, assim fazendo o automonitoramento; a menor frequência dos demais foi associada com a falta de interesse, ausência de fitas na UBS, ou porque só faziam quando solicitado pelo médico ou enfermeiro.

Em relação às consultas diárias, a maioria dos portadores $30(50 \%)$ as realizam a cada 6 meses, e os 12 (20\%) que realizavam mensalmente é devido à necessidade da transcrição da receita para receber os antidiabéticos orais na UBS.

\section{DISCUSSÃO}

A caracterização sociodemográfica dos participantes condiz com outros estudos como o de Sousa JT, et al (2015) e de Corrêa K, et al (2017) nos quais foi identificado o predomínio do sexo feminino, na faixa etária maior de 60 anos. Entretanto, têm-se discordância com o estudo de Santos, AD, et al (2018), em que houve o predomínio do sexo masculino.

Quanto ao grau de conhecimento em relação às práticas de autocuidado, os resultados foram concernentes com o estudo realizado com idosos na atenção primária, que evidenciou que $77,7 \%$ dos diabéticos apresentaram conhecimento insuficiente em relação à sua doença e tratamento (BORBA AKO, et al., 2019). Todavia, entra em discordância com o estudo realizado em um município de Goiânia (GO), no qual $5,4 \%$ dos participantes relataram ausência de conhecimento e $94,6 \%$ conheciam pelo menos uma estratégia para o controle glicêmico (AMARAL RT, et al., 2019).

A percepção sobre as atividades de autocuidado está relacionada ao nível de escolaridade, visto que a falta de ensino e informações afeta o conhecimento dos portadores em relação ao comportamento de autocuidado, pois eles podem apresentar dificuldades para compreender e absorver as recomendações terapêuticas dadas pelos profissionais, o que aumenta a predisposição para complicação e dificulta o enfrentamento da doença. Há, assim, uma necessidade de adaptação de linguagem para cada realidade (SOUSA FDA, et al., 2018).

De Acordo com a teoria de Orem, todos os seres humanos têm potencial para desenvolver habilidades intelectuais e práticas para o autocuidado, que, quando desenvolvidas corretamente, ajudam a manter a integridade estrutural e funcional do corpo. O conhecimento de tais práticas é essencial para o manejo terapêutico, havendo uma necessidade de reconhecimento e ressignificação dessa patologia pelos portadores, integrando-a em sua rotina, em seu ambiente familiar e fora dele (GEORGE JB et al., 2000; TESTON EF, et al., 2017).

Os resultados obtidos em relação as pratica de atividade física concerniram com um estudo realizado em 12 UBS na região nordeste do Brasil, que evidenciou que $62,3 \%$ e $62,5 \%$ dos indivíduos do sexo masculino e feminino, respectivamente, eram sedentários (SOUSA JT, et al., 2015). A prática regular de atividade física reduz a glicemia, pois melhora a sensibilidade à insulina e à captação da glicose, o que contribui para a perca de peso, bem como melhora da função vascular (SOUSA FDA, et al.,2018).

No quesito alimentação saudável houve uma compatibilidade com outros estudos que apresentaram resultados satisfatórios, já que a maioria dos participantes apresentou uma frequência de 5 a 7 dias de uma alimentação saudável (EID LP, et al., 2018; LARRÉ MC, et al., 2018). Diferentemente do estudo realizado com portadores de DM tipo 2 em terapia renal substitutiva, que demonstrou que $61 \%$ seguem uma dieta saudável apenas de 0 a 4 dias e $78 \%$ consomem doces nessa mesma frequência de dias (NOGUEIRA, et al., 2019). 
Alimentação saudável é essencial para o controle glicêmico, pressórico, lipídico e, consequentemente, para a redução do peso corporal, o que minimiza o risco de complicações. O plano alimentar deve atender as necessidades nutricionais do paciente em todas as fases, mas, para isso, o portador deve receber orientações e uma dieta que esteja de acordo com seu contexto cultural e econômico (SOCIEDADE BRASILEIRA DE DIABETES, 2018).

Indivíduos que fazem uso do tabaco têm pior qualidade de vida, e quando associado à DM possui maiores chances de desenvolver complicações, como problemas renais, cardiopulmonar, metabólicos e câncer, pois as células desses portadores possuem maior dificuldade de absorver glicose, provocando maior resistência da insulina. Sua cessação aumenta a sobrevida do portador e melhora o controle glicêmico, reduzindo o risco de complicações (LUCENA AR, et al., 2019). Os resultados foram compatíveis com o estudo realizado em uma UBS no interior de São Paulo, no qual a maioria dos participantes $(91,7 \%)$ se afirmou como não fumante, sendo que apenas $8,3 \%$ faz uso do tabaco (GREGHI EFM e PASCON DM, 2016). Em contrapartida, outro estudo evidenciou que $50 \%$ dos portadores de DM são fumantes (SOUSA JT, et al., 2015).

No tocante à autoexaminação dos pés, os resultados condizem com os do estudo constituído por 163 portadores de DM, o qual demonstrou que $67,5 \%$ têm cuidado com seus pés de 0 a 4 dias na semana, apresentando comportamento de autocuidado indesejável (LARRÉ MC, et al.,2018). Conflitando com a pesquisa realizada com 207 portadores, na qual foi observado que os pacientes realizam cuidado com os pés em média 5 dias na semana, sendo que as mulheres eram mais cuidadosas com a regularidade dessa prática (DANIELE TM, et al., 2014).

O cuidado com os pés é essencial, pois entre as complicações mais comuns está a ocorrência do pé diabético, que pode acarretar danos imensuráveis e dramáticos para a vida do indivíduo, desde feridas crônicas e infecções, até amputações dos membros inferiores, sendo que alguns cuidados são necessários, como higienização, secagem diária, não caminhar descalço, não usar sapatos apertados e fazer sua inspeção, cortar as unhas de forma reta, etc (BRASIL, 2016; LILILANE SP, et al.,2015).

Quanto ao controle glicêmico, os dados corroboram com o estudo realizado no município de Vitória de Santo Antão, Pernambuco (PE), que demonstrou que $87,8 \%$ dos pacientes realizam medidas de glicemia capilar (SANTOS EMS, et al.,2018). O que difere de outro estudo que observou que $75,5 \%$ dos participantes realizam monitorização glicêmica numa frequência de 0 a 4 dias na semana e 24,5\% a realizam de 5 a 7 dias na semana (LARRÉ MC, et al., 2018). Através desse controle diário é possível reduzir de forma significativa o risco de complicações, pois são avaliadas as taxas glicêmicas, assim suputando a magnitude da hiperglicemia ou hipoglicemia, além de servir como parâmetro para o plano alimentar, para as práticas de exercícios e para o tratamento, pois permite ajustes de doses com base nos resultados obtidos diariamente

Quando o portador não consegue o controlar os níveis glicêmico, torna-se predisposto a inúmeras complicações, desde agudas, como cetoacidose, síndrome hiperosmolar hiperglicêmica não cetótica e hipoglicemia, e complicações crônicas, como retinopatia, nefropatia e a neuropatia diabética, além das doenças coronarianas e doença cerebrovascular. O que torna necessário a adoção de hábitos saudáveis, como alimentação balanceada e exercícios físicos, juntamente com os agentes antidiabéticos orais para o controle glicêmico, e, consequentemente, redução das complicações a longo prazo (BRASIL, 2013; DIAS SM, et al., 2018).

No que se refere às consultas diárias, foi observado um desacordo com o estudo realizado com 42 portadores de DM, no qual $31,0 \%$ dos participantes realizam consultas trimestrais e 19,0\%, mensais, existindo ainda outros 19,0\% que não fazem acompanhamento (OLIVEIRA JC, et al., 2018).

Através das consultas é possível estratificar fatores de risco e estabelecer diagnóstico, além de constituir espaço para o incentivo das práticas de autocuidado, discussão do plano terapêutico que converge com a condição econômica e cultural do portador, realização de curativos quando o mesmo apresenta ulceração, monitoramento glicêmico, além de medidas de promoção e prevenção. As consultas devem ocorrer de 3 a 4 meses em portadores com níveis glicêmicos estáveis, e em menor espaço de tempo para aqueles que se encontram descompensados (SANTOS EMS, et al., 2018; QUEIROZ DHS, et al., 2017). 
Quando o indivíduo se encontra em déficit de autocuidado com o que precisa ser feito para restabelecer sua saúde, a enfermagem torna-se necessária para manter as exigências dessas práticas. Tendo em vista esse aspecto, Orem identificou três classificações de sistemas de enfermagem para preencher os requisitos de autocuidado do paciente: sistema totalmente compensatório, que é representado quando o indivíduo é incapaz de provê seu próprio cuidado, o que torna a enfermagem necessária para restabelecer sua saúde; sistema parcialmente compensatório, que é representado por ações de cuidados desempenhadas pelo enfermeiro e paciente; e sistema de apoio e educação, que é quando o indivíduo é capaz de desempenhar ou aprender medidas de autocuidado em benefício próprio, através do apoio da enfermagem. Este apoio é positivo para os portadores de DM que apresentaram um déficit de conhecimento sobre as atividades de autocuidado, o que vai repercutir na clínica da doença (GEORGE JB, et al., 2000).

Todavia, vale enfatizar que o processo de autocuidado é complexo e contínuo, tanto para o paciente, como para o enfermeiro, pois exige decisões e mudanças diárias do estilo de vida frente à patologia; a sua provisão vai de acordo com a realidade e o contexto social que o indivíduo está inserido, pois fatores como falta de conhecimento, baixa autoestima, desmotivação, não aceitação da doença e limitações física e cognitivas podem dificultar esse processo de cuidados (LIMA AA, 2014).

Portanto, o paciente necessita ser orientando pelos profissionais sobre tais ações, em especial pelo profissional da enfermagem que estabelece um maior vínculo com o portador, que influenciará diretamente no manejo clínico da sua doença. Assim, cabendo ao enfermeiro reconhecer os déficits de autocuidado, dialogar com o paciente sobre suas necessidades frente à doença, e estabelecer um plano de cuidado juntamente com o indivíduo, de acordo com suas prioridades, pois as práticas de autocuidado são corresponsabilidade tanto do portador quanto do profissional (TESTON EF, et al.,2017).

O que torna a educação em saúde essencial para a efetivação do tratamento farmacológico como não farmacológico, pois capacita os portadores para o autogerenciamento de sua condição clínica, visto que é a DM é uma doença crônica, cuja grande parte da efetivação do tratamento é construída pela responsabilização e empoderamento do portador, que através de ações educativas adquire conhecimento, habilidades e responsabilidade para a adesão de medidas de autocuidado (IQUIZE RCC, et al., 2017).

\section{CONCLUSÃO}

Foi constatado conhecimento ineficaz dos portadores em relação às atividades de autocuidado, baixa adesão no que se refere à prática de atividade física, cuidado com os pés, monitorização glicêmica e consultas diárias, o que corrobora para o aparecimento de complicações e comorbidades. Porém, quanto a uma alimentação saudável e a não serem tabagistas, apresentaram resultados satisfatórios. É necessário que os serviços de saúde efetuem estratégias de acordo com a realidade da comunidade; com isso, podem utilizarse da teoria do autocuidado de Orem, que objetiva tornar os indivíduos aptos para desempenharem as atividades de autocuidado, afim de manter a funcionalidade do corpo, contribuindo, consequentemente, para o seu bem-estar.

\section{REFERÊNCIAS}

1. AMARAL RT, et al. Conhecimento dos diabéticos frente á doença e orientações no autocuidado. Revista Enfermagem UFPE On Line, 2019; 13(1):346-52.

2. BORBA AKOT, et al. Conhecimento sobre o diabetes e atitude para o autocuidado de idosos na atenção primária à saúde. Revista Ciência e Saúde Coletiva, 2019; 24(1):25-136.

3. BRASIL. Ministério da Saúde. Secretaria de Atenção à saúde. Departamento de Atenção Básica. Caderno de atenção básica no36: Diabetes $2013 . \quad$ Mellitus. >http://bvsms.saude.gov.br/bvs/publicacoes/estrategias_cuidado_pessoa_diabetes_mellitus_cab36.pdf<. Acessado em: 04 abril 2019.

4. BRASIL. Ministério da Saúde. Secretaria de Atenção à Saúde. Departamento de Atenção Básica. Manual do pé diabético: estratégias para o cuidado da pessoa com doença crônica. 2016. Disponível em: http://www.as.saude.ms.gov.br/wp-content/uploads/2016/06/manual_do_pe_diabetico.pdf. Acessado em: 06 de abril de 2019.

5. CORRÊA K, et al. Qualidade de vida e características dos pacientes diabéticos. Revista Ciências \& Saúde coletiva, $2017 ; 22(3): 921-930$. 
6. DANIELE TMC, et al. Avaliação do autocuidado de pacientes com diabetes mellitus tipo 2 em uma unidade de atenção básica. Revista Cinergis, 2014; 15(3):135-139.

7. DIAS SM, et al. Níveis de conhecimento de pacientes diabéticos sobre a Diabetes Mellitus tipo II. Revista Interdisciplinar, 2018; 11(3): 14-21.

8. EID LP, et al. Fatores relacionados às atividades de autocuidado de paciente com Diabetes Mellitus tipo 2. Escola Anna Nery, 2018; 22(4): e20180046.

9. GEORGE JB, et al. Teorias de enfermagem: os fundamentos à prática profissional. 4 ed. Porto alegre: artes medicas, 2000; 83-102.

10. GREGHI EFM, PASCON DM. Conhecimento dos pacientes portadores de diabetes mellitus tipo 2 inseridos no programa de saúde ao adulto. Revista da Faculdade Ciências Médicas de Sorocaba, $2016 ; 18$ (4): 204-9.

11. HINKLE JL, CHEEVER KH. Brunner\&suddarth: Tratado de enfermagem médico cirúrgico. 13 ed. Rio de Janeiro: Guanabara Koogan, 2015; 392p.

12. IQUIZE RCC, et al. Práticas educativas no paciente diabético e perspectiva do profissional de saúde: uma revisão sistemática. Brazilian Journal of Nephrology, 2017; 39(2):196-204.

13. LARRÉ MC, et al. Autocuidado dos pacientes com diabetes mellitus tipo 2 em seguimento ambulatorial. Revista Nursing, 2018; 21(245): 2385-2390.

14. LILIANE SP, et al. Cuidado com os pés: o conhecimento de indivíduos com diabetes mellitus cadastrados no programa saúde da família. Revista Electrónica Trimestral de Enfermería, 2015; (37): 52-62.

15. LIMA AA. O cuidado e o autocuidado de clientes com diabetes e seus familiares: Uso e administração de insulina na Estratégia da Saúde da Família. Trabalho de conclusão de curso (Especialista em Linhas de Cuidado em Enfermagem - Doenças Crônicas Não Transmissíveis (DCNT)) - Universidade Federal de Santa Catarina, Florianópolis, 2014; 33p.

16. LUCENA ACRM, et al. Aspectos facilitadores e dificultadores no abandono do tabagismo entre pessoas com diabetes mellitus tipo 2. Revista Rene, 2019; 23: e-1175.

17. NOGUEIRA BCM, et al. Aspectos emocionais e autocuidado de pacientes com diabetes mellitus tipo 2 em terapia renal substitutiva. Cadernos Brasileiros de Terapia Ocupacional, 2019; 27(1): 127-134.

18. OLIVEIRA JC, et al. Pé Diabético: Perfil sociodemográfico e clínico de pacientes hospitalizados. Revista Brasileira de Ciências de Saúde, 2018; 22(1): 15-20.

19. OLIVEIRA OS, et al. Autocuidado em diabetes mellitus: estudo bibliométrico. Revista Enfermería Global,2017; 16(1): 634-688.

20. PIRES AF, et al. A importância da teoria do autocuidado de Dorothea E. Orem no cuidado de enfermagem. Revista Rede de Cuidados em Saúde. 2015; 9(2).

21. QUEIROZ DHS, et al. A percepção do autocuidado em portadores de diabetes mellitus atendidos na atenção Básica de saúde. Revista Uningá, 2017; 53(2): 12-17.

22. QUEIRÓS PJP, et al. Autocuidado: o contributo teórico de Orem para a disciplina e profissão de enfermagem. Revista Enfermagem Referência, 2014; 4(3): 157-164.

23. SANTOS AD, et al. Perfil epidemiológico de pacientes com diabetes mellitus. Brazilian Journal of Surgery and Clinical Research - BJSCR, 2018; 24(2): 40-46.

24. SANTOS EMS, et al. Autocuidado de Usuários com Diabetes Mellitus: Perfil Sociodemográfico, Clínico e Terapêutico. Revista Cuidado Fundamental, 2018; 10(3): 720-728.

25. SOCIEDADE BRASILEIRA DE DIABETES. Diretrizes Sociedade Brasileira de Diabetes. 2017/2018. Disponível em: https://www.diabetes.org.br/profissionais/images/2017/diretrizes/diretrizes-sbd-2017-2018.pdf. Acessado em: 20 de abril de 2019.

26. SOUSA FDA, et al. Atividades de autocuidado de homens diagnosticados com diabetes mellitus tipo II. Revista Brasileira de Obesidade, Nutrição e Emagrecimento, 2018; 12(76): 1095-1104.

27. SOUSA JT, et al. Autocuidado e parâmetros clínicos em pacientes com diabetes mellitus tipo 2. Revista Rene, 2015; 16(4):479-5.

28. TESTON FE, et al. Perspectivas de indivíduos com diabetes sobre autocuidado: contribuição para assistência. Escola Anna Nery, 2017; 21(2): e20170043. 